\title{
"After-hours" staffing of trauma centres and outcomes among patients presenting with acute traumatic coagulopathy
}

\section{Biswadev Mitra MHSM, PhD, FACEM Emergency Physician \\ Peter A Cameron \\ MBBS, MD, FACEM Head, Critical Care Research² \\ Mark C B Fitzgerald MBBS, FACEM, FRACMA Director, Trauma Services \\ Stephen Bernard MD, FACEM, FCICM Senior Intensive Care Physician \\ John Moloney FANZCA \\ GradCertArts(Writing) GradCertEmergHealth Director, Trauma Anaesthesia \\ Dinesh Varma \\ MBBS, FRANZCR Deputy Director, Radiology \\ Huyen Tran MBBS, FRCPA, FRACP Haematologist \\ Martin Keogh GradDipCCRN GradDipManagement Director, Clinical Services \\ 1 The Alfred Hospital, Melbourne, VIC. \\ 2 Epidemiology and Preventive Medicine Monash University, Melbourne, VIC \\ biswadev.mitra@ monash.edu}

MJA 2014; 201: 588-591 doi: 10.5694/mjal3.00235
. eficiencies in trauma care "after hours" (18:00-07:00) have been well recognised. ${ }^{1}$ Such deficiencies may be caused by the differential availability of senior staff and resources for complex procedures, fatigue of personnel and/ or increased prehospital logistical difficulties (eg, flight restrictions for helicopters at night). Resource allocation and staffing are substantially more expensive after hours, and a finding of an association between time of presentation and outcomes could be used to justify improved staffing in trauma centres outside "business" hours.

Compared with patients admitted "in hours", patients admitted after hours following injury may be intrinsically at higher risk of death by virtue of a different casemix or increased severity of illness. Crude mortality rates have been previously reported to be significantly higher among people admitted with trauma at night, compared with during the day, but no significant association has been shown after adjusting for injury severity. ${ }^{2-5}$

In recent years, mortality after trauma has been steadily decreasing, secondary to preventive strategies and improved trauma systems. ${ }^{6-8}$ Accordingly, in advanced trauma systems, a smaller proportion of inhospital trauma deaths are now preventable. Potential changes to trauma care that could influence mortality therefore affect only a small proportion of the overall trauma population.

Among the overall population of an advanced trauma system, where most deaths are not preventable, the adverse effect of an after-hours model of care may therefore be underestimated. We hypothesise that the effect of any differences in trauma care could be emphasised if patient populations at higher risk of adverse outcomes are analysed.

Patients presenting to the emergency department with acute

Abstract

Objective: To examine the effect of the "after-hours" (18:00-07:00) model of trauma care on a high-risk subgroup - patients presenting with acute traumatic coagulopathy (ATC).

Design, participants and setting: Retrospective analysis of data from the Alfred Trauma Registry for patients with ATC presenting between 1 January 2006 and 31 December 2011.

Main outcome measure: Mortality at hospital discharge, adjusted for potential confounders, describing the association between after-hours presentation and mortality.

Results: There were 398 patients with ATC identified during the study period, of whom 197 (49.5\%) presented after hours. Mortality among patients presenting after hours was $43.1 \%$, significantly higher than among those presenting in hours (33.1\%; $P=0.04$ ). Following adjustment for possible confounding variables of age, presenting Glasgow Coma Scale score, urgent surgery or angiography and initial base deficit, after-hours presentation was significantly associated with higher mortality at hospital discharge (adjusted odds ratio, $1.77 ; 95 \% \mathrm{Cl}$, 1.10-2.87).

Conclusion: The after-hours model of care was associated with worse outcomes among some of the most critically ill trauma patients. Standardising patient reception at major trauma centres to ensure a consistent level of care across all hours of the day may improve outcomes among patients who have had a severe injury.

traumatic coagulopathy (ATC) are at high risk of adverse outcome. ${ }^{9-11}$ Mortality measured at hospital discharge remains high, with most deaths occurring in the first 24 hours. ${ }^{12,13}$ Delayed or suboptimal resuscitation may result in rapid progression of patients to the "triad of death", with worse outcomes. ${ }^{14}$ Therefore, we chose to examine mortality in this clearly defined group of patients with complex injuries, and to examine the effect of the after-hours model of trauma care among them.

\section{Methods}

The state of Victoria, Australia, has one paediatric and two adult major trauma services located in metropolitan Melbourne. Major trauma triage guidelines direct about $85 \%$ of major trauma patients to a major trauma service for definitive treatment. The Alfred Trauma Registry prospectively records prehospital and hospital data on all major trauma patients, defined as those having an Injury Severity Score greater than 15 , requiring urgent surgery or intensive care unit admission, or dying in hospital.

After hours was defined as the period between 18:00 and 07:00, 7 days a week. In the after-hours period, the following specialties were off-site, but on call: emergency physician (02:00-07:00), trauma surgeon (18:00-07:00), intensive care physician (23:00-08:00), haematologist (17:00 07:00) and radiologist (19:00-07:00). The number and seniority of nursing staff in the emergency department and intensive care unit in hours and after hours were similar, but extra staff on administrative duties were present during the day and available to be called on for clinical duties. Nursing staff levels in the operating theatre suite decreased at 18:00, and further at 21:00, to levels facilitating only urgent surgery until 24:00; staff were available on call to open extra theatre capacity, as needed, but with a short delay. The number of laboratory scientists in the haematology laboratory decreased at 17:00 and again at 22:00 until 07:00; again, with potential for recall. 
1 Overall annual inhospital mortality rates (95\% confidence intervals) among patients presenting after major trauma, Alfred Trauma Registry, January 2006 to December 2011

$\diamond \quad$ All patients

- Patients with acute traumatic coagulopathy

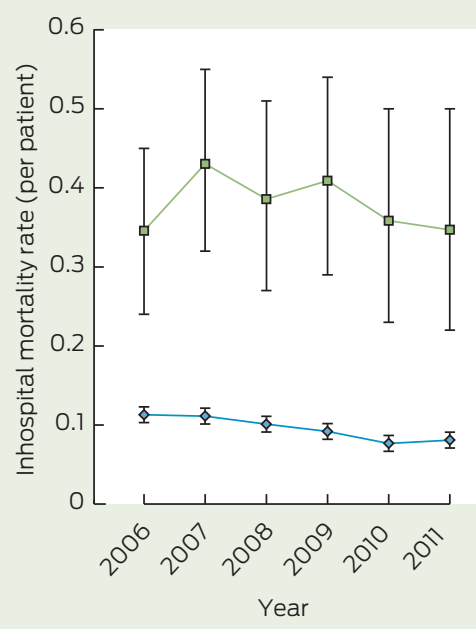

\section{Patients and definitions}

All patients with ATC presenting between 1 January 2006 and 31 December 2011 were included. ATC was defined as an international normalised ratio (INR) greater than 1.5 on the first sample of blood taken after presentation to hospital.11,15,16 Patients receiving anticoagulation treatment were included. A massive transfusion was defined as $\geqslant 5$ units of red cells in the first 4 hours after injury. ${ }^{17}$ Patients who received blood or blood products before presentation were excluded. The shock index was defined as heart rate divided by systolic blood pressure, and the first measured value on presentation was used. ${ }^{18}$ Mortality, as recorded at hospital discharge, was the primary outcome measure.

This study was approved by the Alfred Hospital Research and Ethics Committee.

\section{Statistical analysis}

Normally distributed continuous variables are presented as mean (standard deviation), while ordinal or skewed data are presented as median (interquartile range). The Student $t$ test was used to calculate statistical significance between two means, the Wilcoxon rank-sum test was used for difference between two medians and the $\chi^{2}$ test was used for difference between two proportions.

Results from univariate analyses are reported as unadjusted odds ratios (ORs) with 95\% confidence intervals. Variables exhibiting an association $(P<0.25)$ with the exposure of interest (after hours) and the primary outcome (mortality) were entered into a multivariable logistic regression model to determine the effect of confounders on the association between after-hours presentation and death. ${ }^{19}$ Significance for trends in mortality rates was determined using a Wilcoxon-type test for trend across ordered groups and is reported using $z$ scores. ${ }^{20}$ Results from the multivariable regression model are reported as adjusted ORs with 95\% confidence intervals. All analyses were performed using Stata, version 11.0 (Statacorp).

\section{Results}

During the study period, there were 5915 major trauma presentations, of which 3147 patients (53.2\%) presented after hours. Among all major trauma presentations, inhospital mortality among patients presenting after hours was $9.1 \%$ compared with a mortality of $10.1 \%$ among patients presenting at other times $(P=0.18)$. There were 398 patients $(6.7 \%)$ who presented with ATC. Among all major trauma presentations, there was a significant trend in reduction of mortality over time ( $z$ score, -3.44 ; $P=0.01)$, but the trend in mortality rates among patients with ATC was not statistically significant ( $z$ score, $-0.40 ; P=0.69)$. Overall inhospital mortality rates are shown in Box 1 .

A summary of demographics, vital signs at presentation and injury characteristics of patients with ATC is shown in Box 2. Of the patients with ATC, 197 presented after hours, 85 (43.1\%) of whom died in hospital; and 201 presented in hours, with 67 deaths (33.3\%). After-hours presentation was associated with significantly higher mortality (OR, 1.51; 95\% CI, 1.01-2.28).

A comparison of patients presenting in hours and after hours is shown in Box 3, showing similarly matched groups except for age $(P<0.01)$ and initial base deficit $(P=0.01)$. Variables exhibiting an association between time of presentation and outcome (age, Glasgow Coma Scale [GCS] score, urgent surgery or embolisation and initial base deficit) were entered into a logistic regression model. An independent association between presentation to hospital after hours and significantly higher mortality was observed (adjusted OR, 1.77; 95\% CI, 1.10-2.87). Age (adjusted OR, $1.03 ; 95 \% \mathrm{CI}, 1.02-1.04)$ and presenting GCS score (adjusted OR, 0.80; 95\% CI, $0.77-0.85)$ were also independently associated with mortality. There was no association between requirement for urgent surgery or embolisation (adjusted OR, 1.08; 95\% CI, 0.64-1.84) or initial base deficit (adjusted OR, 0.97; 95\% CI, 0.94-1.01).

After repeating the analysis by including INR categorised into limits, as reported in Box 3, after-hours presentation continued to be associated with higher mortality (adjusted OR, $1.74 ; 95 \%$ CI, 1.07-2.83), along with age (adjusted OR, 1.03; 95\% CI, 1.02-1.04), presenting GCS score (adjusted OR, $0.80 ; 95 \%$ CI, $0.76-0.85$ ) and INR $\geqslant 3.0$ (adjusted OR, 2.05; 95\% CI, 1.03-4.11).

Among patients requiring urgent surgery or embolisation, mean time to theatre or radiology in hours was 3.9 (SD, 3.5), and was not significantly different to the time for patients who underwent urgent surgery or embolisation after hours (mean, 3.7 [SD, 3.7]; $P=0.73$ ). Among patients who received a massive transfusion, the mean ratio of red blood cells to fresh frozen plasma administered in 4 hours was 0.54 (SD, 0.31) after hours and not significantly different to the mean ratio of $0.55(\mathrm{SD}, 0.28)$ in hours $(P=0.85)$. Cryoprecipitate was administered to $0.5 \%$ of patients after hours, compared with $1.5 \%$ in hours $(P=0.32)$.

\section{Discussion}

This study of 398 major trauma patients with ATC shows that a high proportion of such patients present to hospital after hours, when immediately available senior specialist expertise is substantially less. Compared with patients who present in hours, these patients were shown to have significantly higher odds of death when injury severity and demographics were adjusted for. Some aspects of 
2 Characteristics of patients with acute traumatic coagulopathy, Alfred Trauma Registry, January 2006 to December 2011, and univariate associations with mortality at hospital discharge

Variable

Age in years, mean (SD)
Men, no. (\%)
Penetrating mechanism,
ISS, median (IQR)
GCS score, median (IQR)
GCS score < 9, no. (\%)
Shock index in beats per
Shock index $\geqslant 1$, no. (\%)
Positive FAST, no. (\%)
Haemoglobin in g/L, mean
INR, mean (SD)
INR category, no. (\%)
$>1.5-2.0$
$>2.0-2.5$
$>2.5-3.0$
$>3.0$

Base deficit in $\mathrm{mEq} / \mathrm{L}$, mean (SD)

Urgent surgery or angiogram, no. (\%)

Red cell units in 4 hours, median (IQR)

Massive transfusion, no. (\%)

After-hours presentation

\begin{tabular}{ccc} 
Summary & OR $(95 \% \mathrm{Cl})$ of death & $P$ \\
\hline $51.8(24.8)$ & $1.01(0.99-1.02)$ & 0.15 \\
$280(70.4 \%)$ & $1.00(0.64-1.56)$ & 0.99 \\
$20(5.0 \%)$ & $2.55(1.01-6.39)$ & 0.05 \\
$34(25-48)$ & $1.02(1.01-1.04)$ & $<0.01$ \\
$3(3-14)$ & $0.85(0.81-0.88)$ & $<0.01$ \\
$218(54.8 \%)$ & $5.87(3.61-9.54)$ & $<0.01$ \\
$1.87(4.11)$ & $1.04(0.99-1.09)$ & 0.11 \\
$165(41.5 \%)$ & $1.38(0.91-2.09)$ & 0.12 \\
$120(30.1 \%)$ & $0.67(0.42-1.05)$ & 0.97 \\
$106.8(26.9)$ & $0.99(0.98-1.01)$ & 0.09 \\
$2.4(1.0)$ & $1.49(1.21-1.84)$ & $<0.01$
\end{tabular}

$198(49.7 \%)$

$94(23.6 \%)$

$44(11.1 \%)$

$62(15.6 \%)$

$-5.8(7.0)$

$222(55.8 \%)$

$0(0-6)$

$113(28.4 \%)$

$197(49.5 \%)$

$\begin{array}{cc}- & - \\ 1.2(0.7-2.0) & 0.52 \\ 1.4(0.7-2.8) & 0.28 \\ 2.9(1.6-5.2) & <0.01 \\ 0.95(0.92-0.97) & <0.01 \\ 1.69(1.12-2.56) & 0.01 \\ 0.99(0.97-1.01) & 0.28 \\ 0.99(0.63-1.55) & 0.97 \\ 1.51(1.01-2.28) & 0.04\end{array}$

FAST = focused assessment with sonography in trauma. GCS = Glasgow Coma Scale. INR = international normalised ratio. $\mathrm{IQR}=$ interquartile range. ISS = Injury Severity Score. $\mathrm{OR}=$ odds ratio. mortality rates, such as those presenting after traumatic cardiac arrest or those requiring massive blood transfusions. ${ }^{28,29}$ Equally, more senior clinical decision-making capacity onsite would support other key aspects of hospital performance and management of clinical risk.

The challenge for health policymakers is therefore to determine the cost-effectiveness of improved staffing in major trauma centres. Affecting a higher proportion of younger patients, the years of potential life lost because of injury far exceed those of cancer, heart disease, or stroke. Any such change should be accompanied by thorough vigilance to monitor its effect on staff fatigue and potential loss in popularity of critical care specialties. Such adverse effects, if observed, should be weighed against any benefits. In addition, to optimise outcomes, after-hours resources should be enhanced throughout the patients' journeys, from prehospital to the wards or intensive care unit, and not just sporadically in isolated departments.

This study included all patients who presented with ATC to a level 1 adult trauma centre, but is limited in being a retrospective study from a single centre. However, it is worth noting that the after-hours effect size was of substantial magnitude, and higher when adjusted for age, injury severity, GCS score and base deficit. Several confounders were accounted for, but we may have inadequately controlled for casemix or injury severity. Similarly, variables measuring detailed patient management, for example, volumes of fluid and times, ventilator strategies and surgical techniques, were not measured, and are potential confounders. The Trauma and Injury Severity Score ${ }^{30}$ method of severity adjustment was not used, as a large proportion of values for respiratory rates were missing or artificial due to the high frequency of intubated patients. The Injury Severity Score and shock index were used, both of which have been previously validated for use after trauma. ${ }^{31}$

There may be other differences among in-hours trauma patients compared with after-hours, including higher drug and alcohol use or mechanism of injury. Wide variations in the definition of ATC have 
been previously highlighted, 11,32 and it should be noted that repeating this analysis using definitions of lower specificity may dilute the sample with less severely injured patients, limiting validity. Finally, our centre is serviced by a mature prehospital system and is a busy level 1 trauma centre, receiving more than 1200 major trauma patients per year, most of whom are injured by blunt trauma. These results may not be applicable to smaller centres or those with different patient demographics.

Our findings suggest that difference in outcomes observed among patients who presented after-hours was not associated with particular routine processes or timely access to interventions. This generates the hypothesis that such differences could be associated with access to effective coordination by senior decisionmakers during complex resuscitations. Our findings may be applicable to other specialties, and further research is required focused on patients at high risk of adverse outcomes. The decision to prepare for time-critical conditions at all times of day requires quality of outcomes be balanced against the high cost of such care. The feasibility and effectiveness of such change is yet to be determined.

Competing interests: No relevant disclosures.

Received 17 Dec 2013, accepted 02 Jul 2014.

1 Di Bartolomeo S. The "off-hour" effect in trauma care: a possible quality indicator with appealing characteristics. Scand J Trauma Resusc Emerg Med 2011; 19: 33.

2 Carmody IC, Romero J, Velmahos GC. Day for night: should we staff a trauma center like a nightclub? Am Surg 2002; 68: 1048-1051.

3 Busse JW, Bhandari M, Devereaux PJ. The impact of time of admission on major complications and mortality in patients undergoing emergency trauma surgery. Acta Orthop Scand 2004; 75: 333-338.

4 Arbabi S, Jurkovich GJ, Wahl WL, et al. Effect of patient load on trauma outcomes in a leve I trauma center. J Trauma 2005; 59: 815-818; discussion 9-20.

5 Laupland KB, Ball CG, Kirkpatrick AW. Hospital mortality among major trauma victims admitted on weekends and evenings: a cohort study. J Trauma Manag Outcomes 2009; 3: 8.

6 Cameron PA, Gabbe BJ, Cooper DJ, et al. A statewide system of trauma care in Victoria: effect on patient survival. Med J Aust 2008; 189: 546-550.

7 Twijnstra MJ, Moons KG, Simmermacher RK, Leenen LP. Regional trauma system reduces mortality and changes admission rates: a before and after study. Ann Surg 2010; 251: 339-343.

8 Curtis KA, Mitchell RJ, Chong SS, et al. Injury trends and mortality in adult patients with major trauma in New South Wales. Med J Aust 2012; 197: 233-237.
3 Association of variables with time of presentation among patients with acute traumatic coagulopathy, Alfred Trauma Registry, January 2006 to December 2011

Variable

In hours $(n=201)$

Age in years, mean (SD)

$55.8(23.5)$

After hours $(n=197)$

$P$

Men, no. (\%)

$137(68.2 \%)$

47.8 (25.4)

0.01

Penetrating mechanism, no. (\%)

$10(5.0 \%)$

$143(72.6 \%)$

0.33

Blunt mechanism, no. (\%)

$191(95.0 \%)$

$10(5.1 \%)$

$187(94.9 \%)$

ISS, median (IQR)

GCS score,* median (IQR)

34 (25-43)

8 (3-15)

GCS score < 9,* no. (\%)

113 (58.9\%)

1.79 (4.0)

79 (39.7\%)

56 (27.9\%)

106.3 (26.1)

2.4 (1.0)

-4.9 (5.9)

$104(51.7 \%)$

$65(32.3 \%)$

34 (25-50)

3 (3-15)

105 (53.6\%)

1.95 (4.24)

86 (44.3\%)

$64(32.5 \%)$

107.3 (27.7)

$2.4(1.1)$

$-6.7(7.8)$

118 (59.9\%)

48 (24.4\%)

0.96

Urgent surgery or embolisation, no. (\%)

Massive transfusion, no. (\%)

$0(0-8)$

$0(0-4)$

0.86

0.52

0.19

0.29

0.70

0.35

0.31

0.70

0.52

0.01

0.11

0.08

0.06

* Data are missing for this category. FAST = focused assessment with sonography in trauma. GCS = Glasgow Coma Scale.

INR = international normalised ratio. IQR = interquartile range. ISS = Injury Severity Score.

9 Kim SJ, Lee SW, Han GS, et al. Acute traumatic coagulopathy decreased actual survival rate when compared with predicted survival rate in severe trauma. Emerg Med J 2012; 29: 906-910.

10 Gruen RL, Brohi K, Schreiber M, et al. Haemorrhage control in severely injured patients. Lancet 2012; 380: 1099-1108.

11 Brohi K, Cohen MJ, Davenport RA. Acute coagulopathy of trauma: mechanism, identification and effect. Curr Opin Crit Care 2007; 13: 680-685.

12 Mitra B, Cameron PA, Mori A, Fitzgerald M. Acute coagulopathy and early deaths post major trauma. Injury 2012; 43: 22-25.

13 MacLeod JB, Lynn M, McKenney MG, et al. Early coagulopathy predicts mortality in trauma. J Trauma 2003; 55: 39-44.

14 Mitra B, Tullio F, Cameron PA, Fitzgerald M. Trauma patients with the "triad of death". Emerg Med J 2012; 29: 622-625.

15 Kashuk JL, Moore EE, Johnson JL, et al. Postinjury life threatening coagulopathy: is 1:1 fresh frozen plasma: packed red blood cells the answer? J Trauma 2008; 65: 261-270; discussion 270-271.

16 Nardi G, Agostini V, Rondinelli BM, et al. Prevention and treatment of trauma induced coagulopathy (TIC). An intended protocol from the Italian trauma update research group. $J$ Anesthesiol Clin Sci 2013; 2 (1): 22.

17 Zatta AJ, McQuilten ZK, Mitra B, et al. Elucidating the clinical characteristics of patients captured using different definitions of massive transfusion. Vox Sang 2014; 107: 60-70.

18 McNab A, Burns B, Bhullar l, et al. An analysis of shock index as a correlate for outcomes in trauma by age group. Surgery 2013; 154: 384-387.

19 Bursac Z, Gauss CH, Williams DK, Hosmer DW. Purposeful selection of variables in logistic regression. Source Code Biol Med 2008; 3: 17.

20 Cuzick J. A Wilcoxon-type test for trend. Stat Med 1985; 4: 87-90.

21 Luchette F, Kelly B, Davis K, et al. Impact of the in-house trauma surgeon on initial patient care, outcome, and cost. J Trauma 1997; 42: 490-495; discussion 495-497.
22 Helling TS, Nelson PW, Shook JW, et al. The presence of in-house attending trauma surgeons does not improve management or outcome of critically injured patients. J Trauma 2003; 55: 20-25.

23 Reynolds HN, Haupt MT, Thill-Baharozian MC, Carlson RW. Impact of critical care physician staffing on patients with septic shock in a university hospital medical intensive care unit. JAMA 1988; 260: 3446-3450.

24 Arabi Y, Alshimemeri A, Taher S. Weekend and weeknight admissions have the same outcome of weekday admissions to an intensive care unit with onsite intensivist coverage. Crit Care Med 2006; 34: 605-611.

25 Carr BG, Jenkins P, Branas CC, et al. Does the trauma system protect against the weekend effect? J Trauma 2010; 69: 1042-1047; discussion 1047-1048.

26 Schwartz DA, Medina M, Cotton BA, et al. Are we delivering two standards of care for pelvic trauma? Availability of angioembolization after hours and on weekends increases time to therapeutic intervention. J Trauma Acute Care Surg 2014; 76: 134-139.

27 Mitra B, Cameron PA, Gruen RL. Aggressive fresh frozen plasma (FFP) with massive blood transfusion in the absence of acute traumatic coagulopathy. Injury 2012; 43: 33-37.

28 Deasy C, Bray J, Smith K, et al. Traumatic out-of-hospital cardiac arrests in Melbourne, Australia. Resuscitation 2012; 83: 465-470.

29 Mitra B, Mori A, Cameron PA, et al. Massive blood transfusion and trauma resuscitation. Injury 2007; 38: 1023-1029.

30 Boyd CR, Tolson MA, Copes WS. Evaluating trauma care: the TRISS method. J Trauma 1987; 27: 370-378.

31 Olaussen A, Blackburn T, Mitra B, Fitzgerald M. Shock index for prediction of critical bleeding post-trauma: a systematic review. Emerg Med Australas 2014; 26: 223-228.

32 Epstein DS, Mitra B, O'Reilly G, et al. Acute traumatic coagulopathy in the setting of isolated traumatic brain injury: A systematic review and meta-analysis. Injury 2014; 45 : 819-824. 